\title{
ORIGINAL
}

\section{Outcome in patients perceived as receiving excessive care across different ethical climates: a prospective study in 68 intensive care units in Europe and the USA}

D. D. Benoit ${ }^{*} \mathbb{0}$, H. I. Jensen ${ }^{2,3}$, J. Malmgren ${ }^{4}$, V. Metaxa ${ }^{5}$, A. K. Reyners ${ }^{6}$, M. Darmon ${ }^{7}$, K. Rusinova ${ }^{8}$, D. Talmor ${ }^{9}$, A. P. Meert ${ }^{10}$, L. Cancelliere ${ }^{11}$, L. Zubek ${ }^{12}$, P. Maia ${ }^{13}$, A. Michalsen $^{14}$, S. Vanheule ${ }^{15}$, E. J. O. Kompanje ${ }^{16}$, J. Decruyenaere" ${ }^{1}$ S. Vandenberghe ${ }^{17}$, S. Vansteelandt ${ }^{17,18}$, B. Gadeyne ${ }^{1}$, B. Van den Bulcke ${ }^{1}$, E. Azoulay ${ }^{7}$ and R. D. Piers ${ }^{19}$ on behalf of the DISPROPRICUS study group of the Ethics Section of the European Society of Intensive Care Medicine

(c) 2018 The Author(s)

\begin{abstract}
Purpose: Whether the quality of the ethical climate in the intensive care unit (ICU) improves the identification of patients receiving excessive care and affects patient outcomes is unknown.

Methods: In this prospective observational study, perceptions of excessive care (PECs) by clinicians working in 68 ICUs in Europe and the USA were collected daily during a 28-day period. The quality of the ethical climate in the ICUs was assessed via a validated questionnaire. We compared the combined endpoint (death, not at home or poor quality of life at 1 year) of patients with PECs and the time from PECs until written treatment-limitation decisions (TLDs) and death across the four climates defined via cluster analysis.

Results: Of the 4747 eligible clinicians, 2992 (63\%) evaluated the ethical climate in their ICU. Of the 321 and 623 patients not admitted for monitoring only in ICUs with a good $(n=12,18 \%)$ and poor $(n=24,35 \%)$ climate, 36 (11\%) and 74 (12\%), respectively were identified with PECs by at least two clinicians. Of the 35 and 71 identified patients with an available combined endpoint, 100\% (95\% Cl 90.0-1.00) and 85.9\% (75.4-92.0) $(P=0.02)$ attained that endpoint. The risk of death ( $\mathrm{HR} 1.88,95 \% \mathrm{Cl} 1.20-2.92)$ or receiving a written TLD (HR 2.32, Cl 1.11-4.85) in patients with PECs by at least two clinicians was higher in ICUs with a good climate than in those with a poor one. The differences between ICUs with an average climate, with $(n=12,18 \%)$ or without $(n=20,29 \%)$ nursing involvement at the end of life, and ICUs with a poor climate were less obvious but still in favour of the former.
\end{abstract}

Conclusion: Enhancing the quality of the ethical climate in the ICU may improve both the identification of patients receiving excessive care and the decision-making process at the end of life.

Keywords: Perceived excessive care, Ethical climate, Decision-making, Interdisciplinary collaboration, Patient outcomes, Treatment-limitation decisions

\footnotetext{
*Correspondence: dominique.benoit@ugent.be

1 Department of Intensive Care Medicine, Ghent University Hospital, Corneel Heymanslaan 10, Ghent, Belgium

Full author information is available at the end of the article

The full list of investigators of the DISPROPRICUS study group are listed in

the Acknowledgements and in the ESM 3 file.
} 


\section{Introduction}

Life supporting therapy in intensive care units (ICUs) has been increasingly offered to patients with poor longterm prognoses [1,2], including those with advanced, end-stage organ dysfunction or a poor functional status [3-5]. While such therapies should not automatically be considered as non-beneficial, they should be provided only to well-informed patients or relatives in accordance with their preferences and values, and only if treatment intensity remains proportional to the expected outcome $[6,7]$. Nevertheless, one in three deaths occurs during or shortly after ICU treatment [2], frequently following disproportionate levels of care [8-13].

An ethically-based clinical decision-making process has to rely on both individual perceptions and objective criteria, followed by interdisciplinary discussions that enrich the process for the benefit of the patient. However, expressing a perception of excessive care (PEC) to colleagues, and more specifically to senior ones, necessitates a safe climate in which clinicians are empowered to speak up and in which they feel that their opinion is valued and subsequently integrated into the decision-making process [14]. In addition to enhancing trust and cohesion in a team, such a climate may also reduce uncertainty in decision-makers by favoring intra- and interdisciplinary transfer of knowledge, experience and values [14]. Several studies have already shown that concordant prognostic estimates $[15,16]$ or perceptions of inappropriate [17] or futile care [18] by two clinicians may be considerably more predictive about the patient's short- and long-term outcomes than usually thought. However, whether the quality of the ethical climate prevailing in a unit further improves the identification of patients receiving excessive care, and impacts on patient outcomes and written treatment-limitation decision (TLD), is unknown.

The objectives of the current multicenter study were to assess whether the quality of the ethical climate in an ICU is associated with the prognostic value of PEC(s) with regard to patients' one-year outcomes and with the time from PEC(s) until written TLD during ICU stay or death. We hypothesized that the better the ethical climate, the more the PEC(s) would be predictive about patients' one-year outcomes and the shorter the time until written TLD or death.

\section{Methodology}

This study was approved by the ethics committees of all participating centers and the Danish National Health Authority. Informed consent was required in all countries to collect the one-year outcomes. The protocol, questionnaires and case-report form are available in the electronic supplementary material (ESM 1).

\section{Take-home message}

Enhancing the quality of the ethical climate in the ICU may improve both the identification of patients receiving excessive care and the end-of-life decision making process.

\section{Study design and center recruitment}

This 28-day observational study was conducted in 12 European countries and the United States. National coordinators and local investigators were recruited from the Ethics Section of the European Society of Intensive Care Medicine, the APPROPRICUS study group [8] and letters sent to experts in communication and end-of-life care in the ICU. National coordinators were expected to recruit four centers in their country, translate the questionnaires into their own language using the Brislin method [19], obtain ethics committee approval and assist the local investigators in their data collection and data quality tasks. Local investigators arranged study initiation meetings in their ICUs to enhance clinicians' participation, recruited patients after having obtained informed consent and recorded data in a dedicated case-report form on the www.DISPROPRICUS.be website.

\section{Data collection instruments and definition of combined endpoint}

Country, hospital, ICU and clinician characteristics are reported in the ESM 2. Hospital and ICU characteristics were collected by the local investigators between March and May 2014. Country-specific health variables were retrieved from a prior publication [20]. In April and May 2014, clinicians in the participating ICUs completed questionnaires on personal characteristics, working conditions and the ethical climate prevailing in their units using the ethical decision-making climate questionnaire (EDMCQ) [14]. This questionnaire consists of 35 items with four- or five-point Likert scale options; 11 items are on end-of-life care practices; 11 on interdisciplinary reflection, collaboration, and communication and 13 on leadership skills of senior doctors. The theoretical framework and the validation of this instrument can be found in a previous publication [14].

Daily, during the 28 day study period (between May 4 and July 4, 2014), the clinicians anonymously completed a questionnaire about their perceptions of disproportionate care for each of their patients. Disproportionate care was defined as care that is no longer consistent with the expected survival or quality of life (either "too much" or "not enough" care), or that is provided against the patient's or relatives' wishes. Questionnaire completion required less than 5 min per patient per day, when care was perceived as disproportionate, and less than $2 \mathrm{~min}$ 
otherwise. ICU mortality and length of stay were collected in all patients admitted in the ICU; those already admitted prior to the study and those newly admitted during the study period. The characteristics reported in the ESM 2 were collected in patients admitted for reasons other than monitoring only during the study period. Categorization was left at the discretion of the attending physician. Written TLDs were ascertained by chart review.

Because staying at home with a good quality of life is highly valued by patients, the combined patient outcome in this study was defined as dead, not at home or a utility score $<0.5$ at 1 year. This endpoint was defined during a study meeting with the national coordinators at the European Society of Intensive Care Medicine congress in Barcelona on September 30th 2014, approximately one year prior to data collection. Patients admitted for reasons other than monitoring only who were discharged alive, or their families, were contacted by telephone or mail one year after the ICU stay. The interviewer collected vital status, place of residence, and health-related quality of life using the EuroQoL-5D questionnaire [21], with conversion of each health state into a utility index (range -0.1584 to 1.000 ). This questionnaire measures health in five dimensions: mobility, self-care, usual activities, pain/ discomfort, and anxiety/depression. Each dimension has three levels: no problems, moderate problems or severe problems. Therefore, patients can be classified into 1 of 243 possible health states, which is converted into the corresponding utility index (range -0.1584 to 1.000 ), indicating the preference of being in a health status. A utility index $<0.5$ corresponds with severely compromised quality-of-life on at least one of the five dimensions. Although quality-of-life may be preferentially evaluated from the patient, for some older patients proxies may provide the most reliable information [22].

\section{Data analysis}

\section{Ethical climates: factor and cluster analysis}

Using the clinicians' answers to the 35 EDMCQ items, the data were first reduced via exploratory and confirmatory factor analysis to seven latent variables, also called factors [14]. The average score across clinicians for each factor in a given ICU was used as input for the cluster analysis at ICU level (ESM 2). Such analyses seek to minimize the similarity of ICUs within climates and maximize the dissimilarity of ICUs between climates. In particular, we used the partitioning around medoids (PAM) algorithm to classify the different climates into a pre-specified number of clusters. This algorithm was chosen in view of its robustness to outliers and noise [23]. Pearson's chi square tests were used for comparing categorical variables between climates and Kruskal-Wallis tests (or
ANOVA tests where appropriate) for comparing continuous variables. Results were expressed as number (\%) and median (25-75th percentiles), respectively.

\section{Differences in patients' combined endpoint at one year across ethical climates}

To simplify the analysis only perceptions of excessive ("too much") care were taken into account in the current study. As PEC by a clinician alone was only moderately predictive of the patient's combined outcome compared to no PEC across all climates (ESM 2), and previous publications have highlighted the importance of concordance between two clinicians [15-18], we compared the probability of attaining the combined endpoint for patients with PECs by at least two clinicians between the ethical climates. For practical reasons, "PECs by at least two clinicians" is referred to as "concordant PECs" throughout the manuscript. Differences in combined endpoint in patients without and with concordant PECs between and within climates were compared with a Pearson's Chi square and a Fisher's exact test, respectively.

\section{Differences in time until death and treatment limitation decisions across ethical climates}

Time until identification of patients with concordant PECs, and from concordant PECs until written TLD or death were compared using (cause-specific) hazard ratios, obtained via Cox regression (accounting for competing risks) [24]. The cause-specific hazard of an event expresses the instantaneous risk of that event at a given time for patients who are still alive in the ICU at that time and have not previously experienced that event [24]. To better explore the so-called "self-fulfilling prophecy issue" (prognostication influenced by decision-making), we compared the risk of death in patients with concordant PECs in different decision-making scenarios (doctordoctor, doctor-nurse, nurse-nurse) between and within climates.

Adjustment for case-mix, hospital and country characteristics To adjust for differential case-mix, hospital and country characteristics between climates, we used inverse probability weighting based on propensity scores [25]. Here, the propensity score is the probability of being treated in one's own climate, as obtained using a multinomial model based on patient, hospital and country characteristics. Adjustment based on propensity scores has the advantage, relative to other adjustment methods, of preventing model extrapolation, when climates are very different in terms of these characteristics [25]. However, one concern about adjustment for case-mix is that it may eliminate the effects of potential differences in admission policy (which affects case-mix) between climates. 
Therefore, we considered the unweighted results as our principal results. These are expressed as proportions and (cause-specific) hazard ratios (HR) along with 95\% confidence intervals $(95 \% \mathrm{CI})$. Two-sided $P$ values were considered significant at the 0.05 level. Priority was given to comparisons between the good and the poor ethical climates (see results) in order to reduce type I errors. We refer to the ESM 2 for a more detailed methodology.

\section{Results}

\section{Ethical climates}

Of 4747 clinicians working in 68 ICUs in Belgium, Czech Republic, Denmark, France, Germany, Greece, Hungary, Italy, Portugal, United Kingdom, Sweden, the Netherlands and the United States, 2992 (62.6\%) completed the EDMCQ (Fig. 1).

The cluster analysis based on the average scores of the seven factors identified during the validation of the EDMCQ [14] yielded four different meaningful, mutually exclusive ethical climates. Visual inspection of the scree plot (ESM 2) revealed that clustering into three clusters would drastically increase the total intra-cluster variation (as opposed to using four clusters), while clustering into five clusters would only minimally decrease the total intra-cluster variation [23]. These climates were denominated by experts in intensive care (DB, JD), psychology (BV, SV) and ethics (RP) as: good, average with ${ }^{(+)}$and without $^{(-)}$nurses' involvement at end-of-life, and poor (Fig. 2, ESM 2). According to clinicians working in a good climate, leadership by senior doctors is active and facilitates interdisciplinary reflection and decision-making overall. This climate is also characterized by mutual respect, which is pre-requisite to facilitating interdisciplinary reflection and ethical awareness [14]. Within the average $^{(+)}$climate, clinicians perceive that senior doctors empower nurses to share interdisciplinary decision-making, mainly at end-of-life. Even though clinicians working in an average ${ }^{(-)}$climate believe that their senior doctors are able to make decisions, they do not find them promoting nurse involvement in decision making at end-oflife. Finally, clinicians working in a poor climate perceive a need for improvement in all of these factors.

The ICU, clinician, and patient characteristics for each climate are reported in ESM 2. The average ${ }^{(-)}$and poor climates were more prevalent in Central and Southern European countries $(P<0.001)$; however, 10 of the $24(41.7 \%)$ ICUs with a poor climate were situated in Western Europe and the United States. The ICU experience of clinicians was similar across climates, however, the number of participating doctors was higher in the average $\mathrm{e}^{(-)}$and poor, compared to the other two climates. The average $e^{(-)}$and poor climates were also associated with a slightly higher number of admitted patients with severe underlying comorbidities and with greater use of advanced and prolonged life-supporting treatments in the post-operative setting, compared to the other climates.

\section{Differences in patients' combined endpoint at one year across ethical climates}

Of the 1761 patients admitted for more than only monitoring with data concerning time until event available (Fig. 1), 74 (4.2\%) patients were perceived as receiving excessive care by two clinicians, and $107(6.1 \%)$ by more than two clinicians, resulting in 36 (11.0\%), 50 (7.2\%), 21 $(18.0 \%)$ and $74(12.0 \%)$ patients with concordant PECs from the good to the poor climate, respectively. Excessive care was perceived by these clinicians as being provided against the patients' or relatives' wishes in 20 (55.5\%), $25(50.0 \%), 11(52.4 \%)$ and $41(55.4 \%)(P=0.94)$ of these patients.

The differences in the patients' combined outcomes across ethical climates are reported in Table 1. The probabilities of attaining the combined endpoint in patients without concordant PECs was 53.5\% (95\% CI 46.8-60.2), $59.1 \%$ (54.6-63.6), 64.0\% (53.1-74.9) and 51.8\% (47.356.3) from good to poor climate, respectively $(P=0.057$, difference between good and poor climate, $P=0.74$ ). These probabilities increased to $100 \%(90.0-100), 95.6 \%$ (84.3-98.9), 94.7\% (70.6-99.3) and 85.9\% (75.4-92.0) in patients with concordant PECs $(P=0.047$, difference between good and poor climate, $P=0.020$ ).

\section{Differences in time until death and treatment limitation decisions across ethical climates}

We found no difference in incidence or in time from admission until concordant PECs between the good and the poor climates; approximately $11 \%$ of the patients were identified with concordant PECs after 14 days in both climates (Fig. 3a).

The risk of death in patients with concordant PECs was statistically significantly higher ( $\mathrm{HR} 1.88,95 \% \mathrm{CI}$ 1.20-2.92) in the good compared to the poor climate. The median time until death in patients with concordant PECs was 5 days $(2-18)$ vs. $14(6-34)$ days $(P=0.008)$, respectively. The difference between the average climates and the poor climate was less important, but still in favor of the average climates (Fig. 3c). The risk of death in the good climate was higher in patients with PECs by two or more doctors than in those with PECs by two or more nurses (HR 3.13, 95\% 1.19-8.23), with the risk of death in patients with PECs by at least one nurse and one doctor being intermediate. There was no evidence of such a difference in risk of death in the poor climate (HR 0.74, 95\% 0.29-1.86) (ESM 2). 


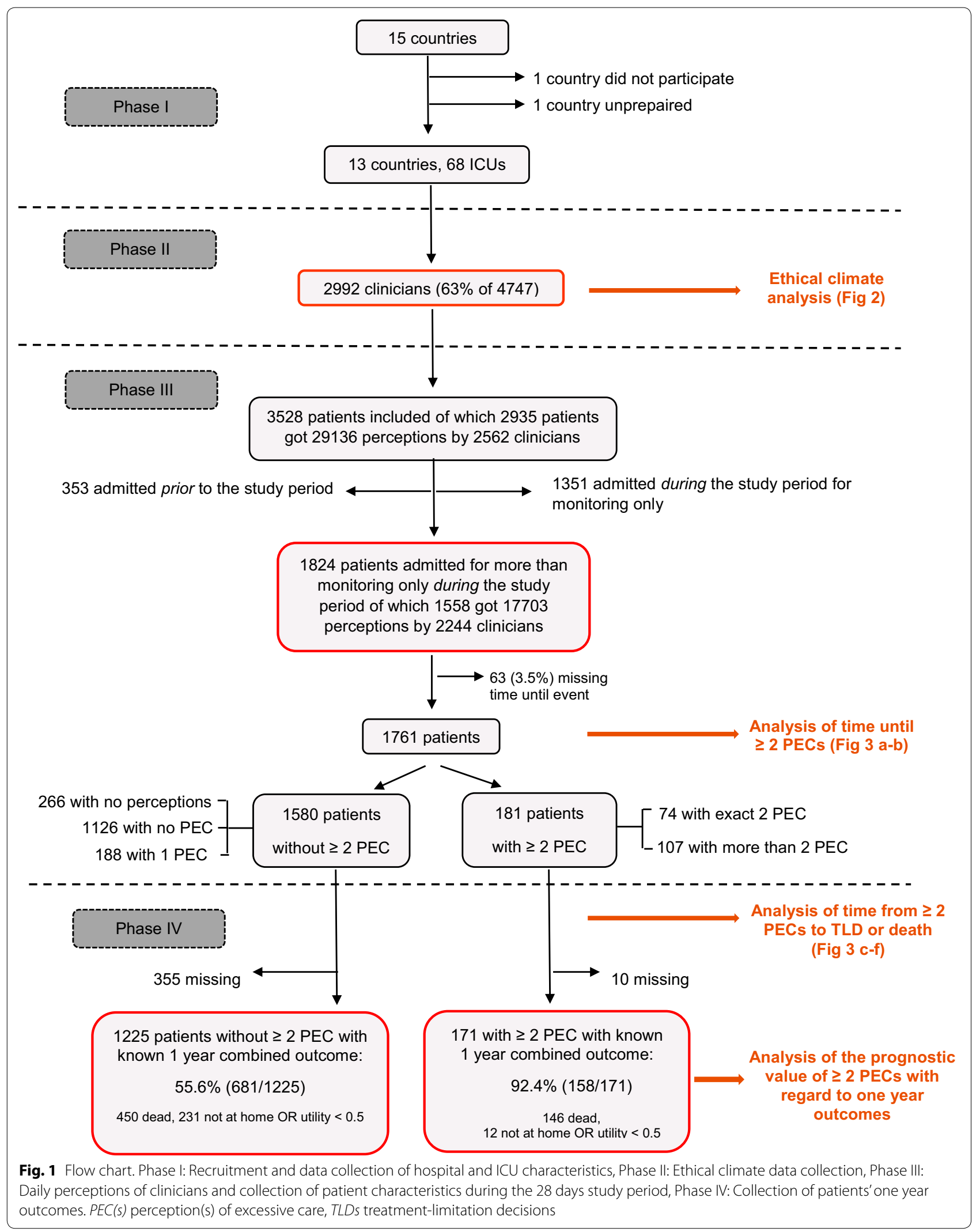




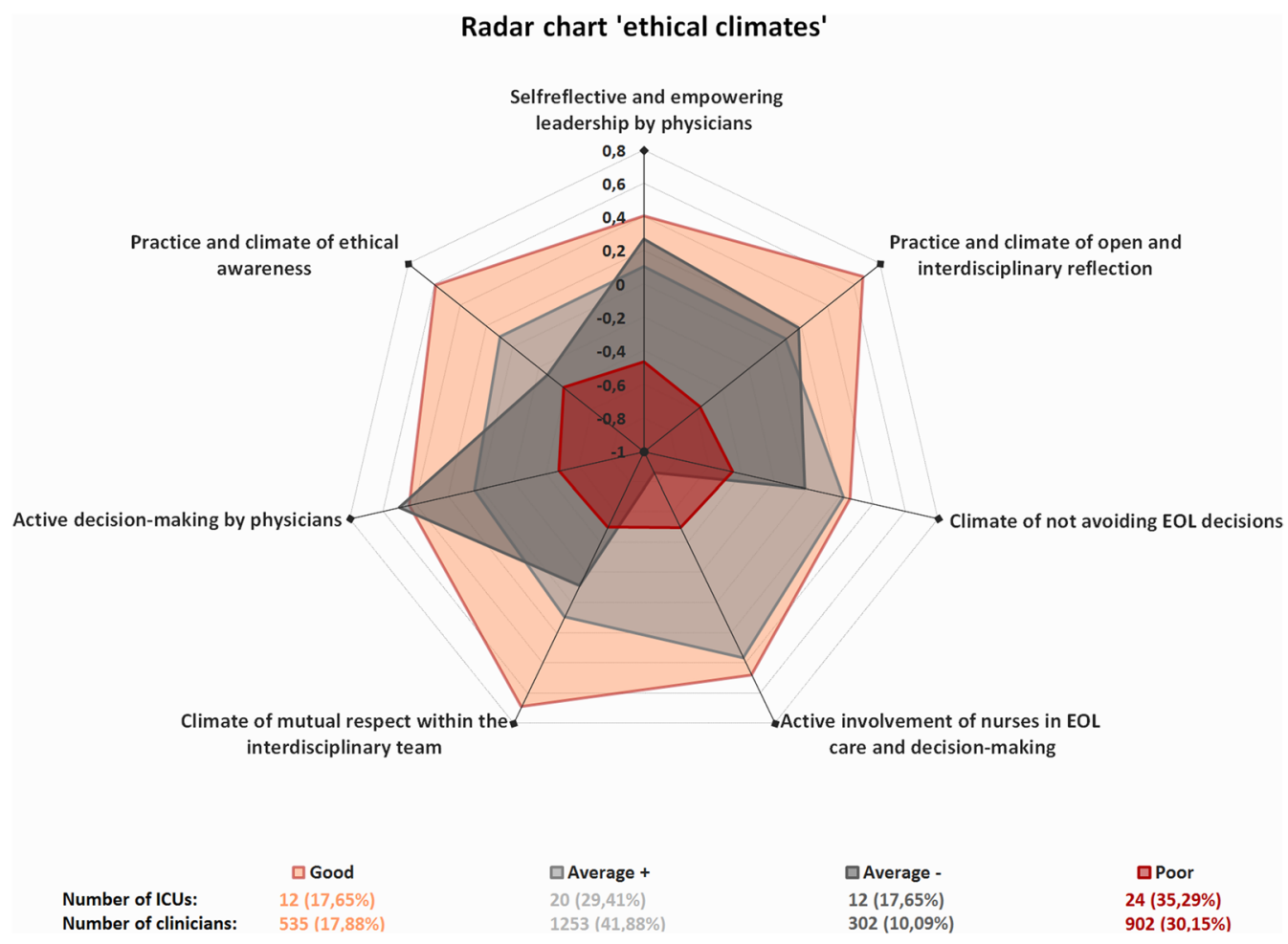

Fig. 2 Ethical climates. Factor and cluster analysis were used to obtain mutually exclusive climates. Factor analysis attributes and aggregates the 35-item ethical decision-making climate questionnaire into seven factors for each clinician, which describe different aspects of the ethical decisionmaking climate as perceived by that clinician. These were subsequently averaged across clinicians to obtain seven factor scores per ICU [14]. A cluster analysis based on these averages scores identified four meaningful ethical climates; good, average with ${ }^{(+)}$and without ${ }^{(-)}$involvement of nurses at end-of-life (EOL), and poor. The figure visualizes the average factor scores in clinicians per climate. Larger values indicate better agreement with the climate expressed by the corresponding factor. More detailed information can be found in the ESM 2

Table 1 Differences in patients' one-year outcomes across ethical climates in patients with and without concordant PECs

\begin{tabular}{|c|c|c|c|c|c|c|}
\hline & \multicolumn{4}{|c|}{ Ethical climate } & \multirow{2}{*}{$P$ value overall } & \multirow{2}{*}{$P$ value good vs. poor climate } \\
\hline & Good & Average $^{(+)}$ & Average $^{(-)}$ & Poor & & \\
\hline Patients without concordant PECs $(n=1225)$ & $n=215$ & $n=464$ & $n=75$ & $n=471$ & & \\
\hline Combined endpoint $^{a}$ & $115(53.5 \%)$ & $274(59.1 \%)$ & $48(64.0 \%)$ & $244(51.8 \%)$ & 0.057 & 0.740 \\
\hline Dead & $68(31.6 \%)$ & $175(37.8 \%)$ & $39(52.0 \%)$ & $168(35.7 \%)$ & & \\
\hline Alive not at home or utility $<0.5$ & $47(21.9 \%)$ & $99(21.3 \%)$ & $9(12.0 \%)$ & $76(16.1 \%)$ & & \\
\hline Patients with concordant PECs $(n=171)$ & $n=35$ & $n=46$ & $n=19$ & $n=71$ & & \\
\hline Combined endpoint ${ }^{b}$ & $35(100 \%)$ & $44(95.6 \%)$ & $18(94.7 \%)$ & $61(85.9 \%)$ & 0.047 & 0.020 \\
\hline Dead & $33(94.3 \%)$ & $41(89.1 \%)$ & $18(94.7 \%)$ & $54(76.0 \%)$ & & \\
\hline Alive not at home or utility $<0.5$ & $2(5.7 \%)$ & $3(6.5 \%)$ & $0(0.0 \%)$ & $7(9.9 \%)$ & & \\
\hline
\end{tabular}

After weighting to adjust for differential case-mix, hospital and country characteristics, the probability of attaining the combined endpoint in patients ${ }^{\text {a }}$ without and ${ }^{b}$ with concordant PECs was $56,62,60$ and $55 \%(P=0.26$, difference between good and poor climate, $P=0.82)$ and $100,93.9,93.5$ and $86.2 \%(P=0.042$, difference between the good and the poor climate, $P=0.017)$ from the good to the poor climate, respectively

Patients with concordant PECs had a higher chance of receiving a written TLD in the good compared to the poor climate (cause-specific HR 2.32, 95\%CI 1.11-4.85) (Fig. 3e).

\section{Adjustment based on propensity scores}

After weighting to adjust for differential case-mix, hospital and country characteristics, the probability of attaining the combined endpoint in patients without 


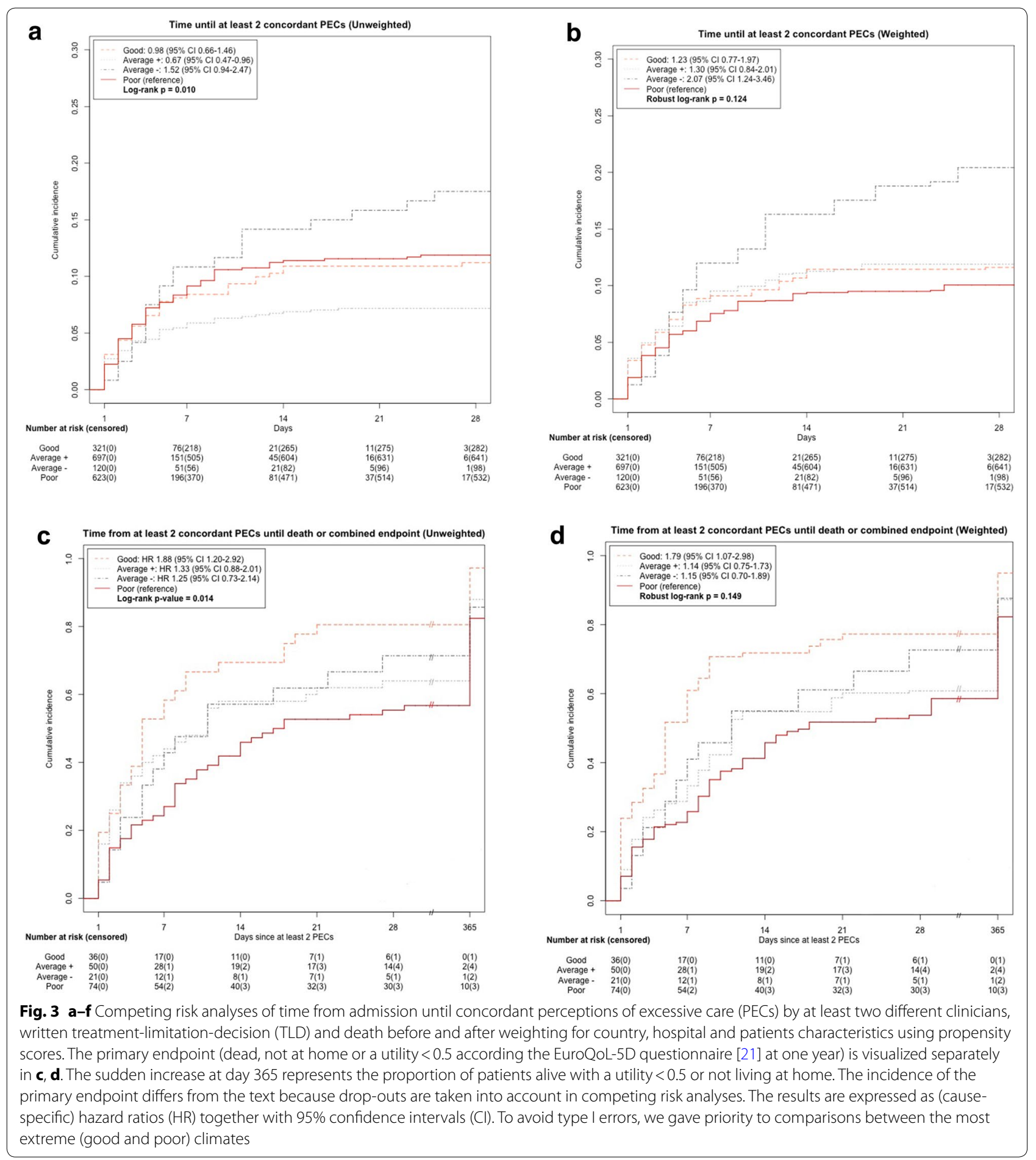

concordant PECs was 55.8\% (48.2-63.1), 62.1\% (56.5$67.4), 60.2 \%(47.4-71.7)$ and $54.8 \%(49.4-60.1)$ from good to poor climate, respectively $(P=0.26$, difference between good and poor climate, $P=0.82$ ). These probabilities increased in patients with concordant PECs to

100\% (90.0-100), 93.9\% (74.3-98.8), 93.5\% (64.2-99.1) and $86.2 \%$ (72.0-93.8), respectively $(P=0.042$, difference between the good and the poor climate, $P=0.017$ ). The risk of death in patients with concordant PECs also remained higher in the good vs. the poor climate 

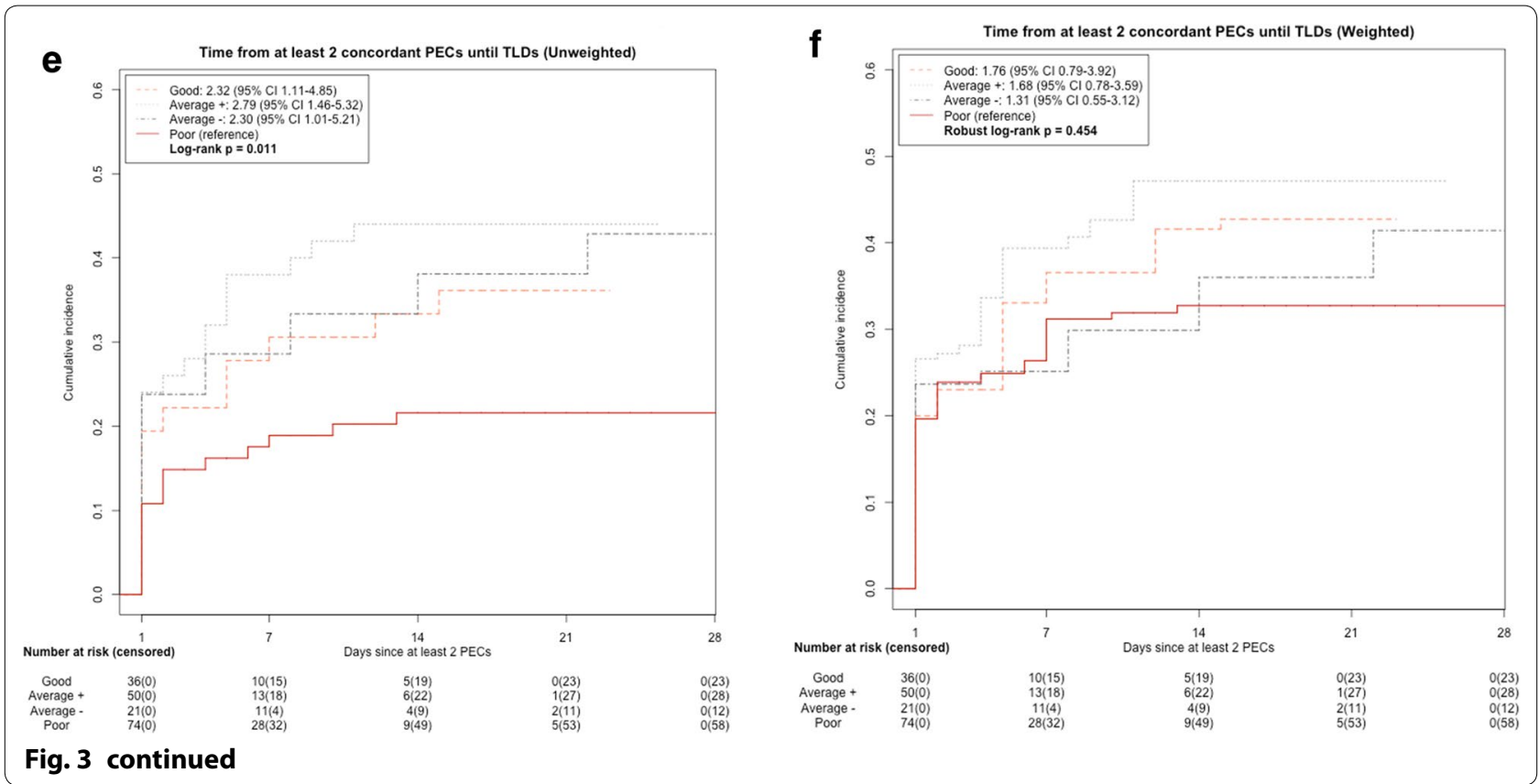

(HR 1.79, 95\%CI 1.07-2.98) (Fig. 3d). The median time until death was $5(2-18)$ and $14(7-30)$ days $(P=0.026)$, respectively. The risk of death in the good climate remained higher in patients with PECs by two or more doctors than in those with PECs by two or more nurses (HR 3.58, 95\% 1.42-9.02), with the risk of death in patients with PECs by at least one nurse and one doctor remaining intermediate. There was no evidence of such a difference in risk of death in the poor climate (HR 1.58, 95\% 0.45-5.55) (ESM 2).

However, we no longer found evidence of a difference in time until TLD between the good and the poor climates (cause-specific HR 1.76, 95\%CI 0.73-3.92) (Fig. 3f).

\section{Discussion}

In this large, multicenter, prospective, ICU study, we found that concordant PECs by at least two clinicians were far more predictive about the primary composite endpoint of death, not living at home, or having poor quality of life one year after ICU admission, compared to absence of PEC. We found evidence of a difference in one-year outcomes, time until death and written TLD in patients with concordant PECs across the four ethical climates identified by our questionnaire. The evidence of a difference in time until written TLD disappeared after adjusting for differential case-mix, hospital and country characteristics.

In contrast to the study by Detsky et al. [16], clinicians in our study were not explicitly expected to provide prognostic estimates about the patients' outcomes.
We preferred to focus on the intuitive-heuristic more than the analytic-deductive part of the complex ethical decision-making process [26, 27], by asking clinicians whether they felt that the care provided to their patient on a specific day was consistent with the expected outcome in terms of survival and quality of life, and whether this amount of care was in line with the patient's or relatives' wishes. We also didn't focus on futile care, such as in the studies of Neville et al. [18], because this terminology presupposes a high degree of certainty concerning the final fatal prognosis, whereas nowadays technological innovation frequently excludes patients' spontaneous death in ICU $[6,7]$. By doing so, we acknowledged uncertainty [26] (benefit vs. harm) and patient and family autonomy, as an integral part of the complex ethical decision-making process at the bedside [28]. Nevertheless, PEC was highly predictive about patients' one-year outcomes, more specifically when expressed by two or more than two clinicians.

Concordant PECs by at least two different clinicians were more predictive about the combined endpoint in the good compared to the poor ethical climate $(P=0.028)$. Patients with concordant PECs also had a higher risk of death and of receiving a written TLD in the good compared to the poor climate. The difference in endpoints between the average and the poor climates was less obvious, but still in favor of the former compared to the latter, thus objectively validating our EDMCQ instrument [14]. Unfortunately, we can neither exclude nor confirm self-fulfilling prophecy in the good climate. However, it is of note that it took about 14 days 
to identify all patients with concordant PECs in both climates and, for half of these patients, another 5 days to die in the good vs. 14 days in the poor climate $(P=0.002)$. In line with the results of the EDMCQ, this suggests that the decision to forgo life sustaining treatment in the good climate was not premature, and once excessive care was perceived by at least two clinicians, it occurred in a timely fashion. Furthermore, in a sub-analysis, we found no difference in risk of death between patients with concordant PECs by different professionals in the poor climate, as opposed to the good climate. This indicates that identification of patients with excessive care by doctors in the poor climate was not followed by active decisionmaking. In addition to, respectively, increasing the risk of prolonged suffering and complicated grief in patients and relatives [29,30], decision-paralysis as a strategy to cope with prognostic uncertainty $[8,12,31]$ may also induce moral distress and increase intention to leave in clinicians [6, 32-34]; a fact that is even more pertinent considering the high number of concordant PEC records perceived as violating the patient's or relatives' wishes in this study. After weighting for the specific case-mix within a hospital and country, only the risk of receiving a written TLD in the good compared to the poor climate was no longer significantly different. This may suggest that the quality of the ethical climate in an ICU is important in identifying patients receiving excessive care and in subsequently triggering the decision-making process at end-of-life, whereas formalizing that process via a written TLD seems more case-mix and culture dependent. This is in line with previous studies showing a huge variability in written TLDs between countries and ICUs [35].

The probability of dying or surviving with a poor quality of life at one year in patients without concordant PECs was 53.5, 59.1, 64.0 and 51.8\% from good to poor climate, respectively, largely exceeding that of many malignancies [36]. Therefore, in line with the definition of disproportionate care $[6,8,9]$, clinicians did not find poor prognosis sufficient by itself to lead to a PEC. Concordant PECs by at least two clinicians increased the probability of reaching the combined endpoint to $100 \%$ in the good, $95.6 \%$ in the average ${ }^{(+)}$and $94.7 \%$ in the average ${ }^{(-)}$climate, compared to $85.9 \%$ in the poor. Despite the poor prognosis we found a relatively low incidence of written TLDs within the 14 days in these patients; ranging from $20 \%$ in the poor to only $35 \%$ in the good and about $45 \%$ in average climates $(P=0.011)$. Although caution in interpreting this result is required due to small sample size, these probabilities highlight the urgent need for improving advance-care planning before ICU admission [37], as well as triage and decision-making at end-of-life in ICU. This should more specifically be achieved via ethical climates that favor interdisciplinary reflection and collaboration $[6,8,14,32,38,39]$, and early involvement of palliative care [30, 37, 40]. Our EDMCQ instrument may be used for that purpose $[14,32]$.

Our study has several limitations. First, the participating ICUs were not selected at random, which may have affected the external validity of our results. Second, inclusion of patients was left at the discretion of the attending doctor. However, except in the average ${ }^{(-)}$climate (ESM 2), we found no evidence of a difference in ICU mortality rates or length of stay in the subgroup of patients admitted for monitoring only across climates, indicating that the attending doctors included patients in a similar way. We further minimized confounding bias by accounting for differences in case-mix, using inverse probability weighting based on propensity scores. Third, we did not use classical severity-of-illness scores in our analysis. However, in line with our primary objective, we preferred to include short- and long-term prognostic factors $[4,5]$ that are commonly used by clinicians during decisionmaking, rather than classical severity-of-illness scores which have never been validated for predicting longterm outcomes. Fourth, one has to keep in mind that the incidence of patients with concordant PECs is probably underestimated, as patients admitted prior to the study period and those who remained in ICU for longer than the study period (and were expected to reach more clinician concordance with time) were excluded from the analysis. Finally, although the ICU experience of clinicians was similar, we cannot exclude that the lower number of participating doctors in the good compared to the poor climate may have biased our results in favor of the latter, concealing even larger differences between the two.

\section{Conclusion}

Our results suggest that improving the quality of the ethical climate in ICU may favor the identification of patients receiving excessive care and the subsequent decisionmaking process at end-of-life. This may benefit the quality of the dying process in ICUs.

\section{Electronic supplementary material}

The online version of this article (https://doi.org/10.1007/s00134-018-5231-8) contains supplementary material, which is available to authorized users.

\footnotetext{
Author details

${ }^{1}$ Department of Intensive Care Medicine, Ghent University Hospital, Corneel Heymanslaan 10, Ghent, Belgium. ${ }^{2}$ Department of Intensive Care Medicine, Vejle Hospital, Vejle, Denmark. ${ }^{3}$ Institute of Regional Research, University of Southern Denmark, Odense C, Denmark. ${ }^{4}$ Department of Anaesthesiology and Intensive Care, Sahlgrenska University Hospital, Gothenburg, Sweden. ${ }^{5}$ King's College Hospital, London, UK. ${ }^{6}$ Department of Medical Oncology, University of Groningen, University Medical Center Groningen, Groningen, The Netherlands. ${ }^{7}$ Hôpital Saint-Louis and University, Paris-7, Paris, France. ${ }^{8}$ Department of Anesthesiology and Intensive Care, First Faculty of Medicine, Charles University in Prague and General University Hospital in Prague, Prague, Czech Republic. ${ }^{9}$ Department of Anesthesia, Critical Care, and Pain Medicine, Beth Israel Deaconess Medical Center and Harvard Medical School, Boston,
} 
MA, USA. ${ }^{10}$ Service des soins intensifs et urgences oncologiques, Institut Jules Bordet, ULB, Brussels, Belgium. " ${ }^{11}$ SCDU Anestesia e Rianimazione, Azienda and Ospedaliero Universitaria, "Maggiore della Carità", Novara, Italy. ${ }^{12}$ Semmelweis University Budapest, Budapest, Hungary. ${ }^{13}$ Intensive Care Department, Hospital S.António, Porto, Portugal. ${ }^{14}$ Tettnang Hospital, Tettnang, Germany. ${ }^{15}$ Department of Psycho-analysis and Clinical Consulting, Faculty of Psychology and Educational Sciences, Ghent University, Ghent, Belgium. ${ }^{16}$ Department of Intensive Care Medicine, Erasmus MC University Medical Center Rotterdam, Rotterdam, The Netherlands. ${ }^{17}$ Department of Applied Mathematics, Computer Science and Statistics, Faculty of Sciences, Ghent University, Ghent, Belgium. ${ }^{18}$ London School of Hygiene and Tropical Medicine, London, UK. ${ }^{19}$ Department of Geriatric Medicine, Ghent University Hospital, Ghent, Belgium.

\section{Acknowledgements}

This study was supported by a European Society of Intensive Care Medicine/European Critical Care Research Network clinical research award and a Fonds voor Wetenschappelijk Onderzoek senior clinical investigators grant (1800513N) obtained in 2012 by DB. We are grateful to Ariella Van Sompel for having performed the factor and cluster analysis together with VDB and RP (under supervision of SVH and SVS) and Jolien Roels for having performed the data cleaning and the univariate analysis (under supervision of DB, SVB and SVS). Participating centers and local investigators: Belgium: University Hospital, Vrije Universiteit Brussel, Brussels (Herbert Spapen, Marie-Claire Van Malderen, Godelieve Opdenacker), Leuven University Hospital, Leuven (Geert Meyfroidt, Dieter Mesotten, Joost Wauters, Marie Van Laer and Alexander Wilmer, Joost Wauters, Helga Ceunen), ZNA Stuivenberg, Antwerpen (Inneke E De Laet, Anita Jans), Ghent University Hospital, Gent (Dominique Benoit, Sandra Oeyen, Ingrid Herck, Stephanie Bracke, Charlotte Clauwaert), Institut Jules Bordet, Bruxelles (Meert Anne-Pascale, Leclercq Nathalie), CHU-Brugmann, Bruxelles (Devriendt Jacques), CHU Saint Pierre, Bruxelles (Dechamps Philippe), Czech Republic: Liberec District Hospital, Liberec (Ivana Zykova), Masaryk University, Brno and University Hospital, Brno (Jan Malaska), Third Faculty of Medicine, Charles University, Prague (Matous Schmidt), Hospital and Polyclinic Havirov, Havirov (Igor Satinsky), Institute for Experimental and Clinical Medicine, Prague (Eva Kieslichova), 3rd Medical Department, First Faculty of Medicine, Charles University in Prague and General University Hospital, Prague (Jarmila Krizova), Karlovy Vary District Hospital, Karlovy Vary (Robert Janda), Pardubice District Hospital, Pardubice (Magdalena Fortova, Jiri Matyas), First Faculty of Medicine, Charles University and General University Hospital, Prague (Katerina Rusinova, Ondrej Kopecky), Denmark: Herning Hospital, Herning (Christian Alves Køhler Pedersen), Kolding Hospital, Kolding (Stine Hebsgaard), Vejle Hospital, Vejle (Rikke Frank Aagaard Johnsen), Holbæk Hospital, Holbæk (Tina Charlotte Bitsch Hansen), France: Saint-Etienne University Hospital and Jacques Lisfranc Medical School, Saint-Etienne (Michael Darmon), Saint-Louis University Hospital, APHP, Université Paris-7, Paris (Danielle Reuter, Elie Azoulay), Institut Paoli Calmette, Marseilles (Djamel Mokart), Montfermeil Hospital, Montfermeil (François Vincent), Germany: University Hospital Jena, Jena (Christiane S. Hartog), Viersen General Hospital, Viersen (Peter Gretenkort), Tettnang Hospital, Tettnang (Andrej Michalsen), Greece: Agia Olga Hospital, Athens (Aikaterini Kounougeri), Evangelismos Hospital, Athens (Serafim Nanas), Agios Pavlos Hospital, Thessaloniki (Despina Papachristou), AHEPA University Hospital, Thessaloniki, (loanna Soultati), G.Gennimatas Hospital, Thessaloniki (Dimitrios Lathyris), Hippokratio General Hospital, Thessaloniki (Marili Pasakiotou), Papageorgiou General Hospital, Thessaloniki (Marina Oikonomou), Hungary: Semmelweis University Budapest, Budapest (Gábor Élő, Orsolya Szűcs), Kaposi Mór Teaching Hospital, Kaposvár University, Kaposvár (János Fogas), St. Stephen and St. Leslie Metropolitan Hospital, Budapest (llona Bobek), Italy: Azienda Ospedaliero Universitaria, "Maggiore della Carità", Novara, and Department of Translational Medicine, Università del Piemonte Orientale, Novara (Francesco Della Corte, Carlo Olivieri, Rosanna Vaschetto, Laura Cancelliere), Ospedale Civile San Salvatore, and Department of Life, Health and Environmental Sciences (MeSVA), University of L'Aquila and Department of Emergency, San Salvatore Hospital, L'Aquila (Franco Marinangeli, Tullio Pozone, Alessandra Ciccozzi), The Netherlands: Canisius Wilhelmina Ziekenhuis, Nijmegen (A. Schouten, Monique Bruns), Medical Center Leeuwarden, Leeuwarden (Rik T. Gerritsen, Matty Koopmans), Erasmus University Hospital of Rotterdam (Erwin Kompanje, Ditty van Duijn), University of Groningen and University Medical Center Groningen, Groningen (Jan G. Zijlstra, Anne KL Reyners), Wilhelmina Ziekenhuis Assen, Assen (Johan G. Lutisan), Portugal: Hospital S.António, Porto (Raquel Monte, José António Pinho, Pedro Pimenta), CHVNG, Vila Nova de
Gaia (Paula Fernandes, Ana Isabel Paixão), Instituto Português de Oncologia, Porto (Filomena Faria), Sweden: Sahlgrenska University Hospital, Gothenburg (Johan A. Malmgren), Sahlgrenska University Hospital/Östra, Gothenburg (Bertil Andersson), Skåne University Hospital, Malmö (Eva Åkerman), Karolinska University Hospital, Karolinska (Andreas Hvarfner), The Hospital of Norrköping, Norrköping (Robert Svensson), United Kingdom: King's College Hospital, London (Victoria Metaxa), USA: Beth Israel Deaconess Medical Center and Harvard Medical School, Boston MA (Daniel Talmor, Ariel Mueller, Valerie Banner-Goodspeed), Henry Mayo Newhall Memorial Hospital, Valencia, CA (Dee Rickett), Mayo Clinic, Rochester, MN (Michael E. Wilson, Richard Hinds).

\section{Author Contributions}

Study concept and design: DDB, BVB, RDP. Design of the questionnaire: DDB, $H I J, J M, ~ S V, E J O K, J D, B V B, E A, R D P$. Coordination of the translation of the questionnaire: HIJ, JM, VM, AKR, MD, KR, DT, APM, LC, LZ, PM, AM. Acquisition of data: DDB, HIJ, JM, VM, AKR, MD, KR, DT, APM, LC, LZ, PM, AM, BG. Analysis and interpretation of data: DDB, SV, SV, SV, BVB, EA, RDP. Drafting of the manuscript: $\mathrm{DDB}, \mathrm{VM}, \mathrm{DT}, \mathrm{SV}, \mathrm{BVB}, \mathrm{EA}, \mathrm{RDP}$. Critical revision of the manuscript for important intellectual content: DDB, HIJ, JM, VM, AKR, MD, KR, DT, APM, LC, LZ, PM, AM, SV, EJOK, JD, SV, SV, BG, BVB, EA, RDP. Statistical expertise: SV, SV. Obtained funding: DDB, JD. Administrative, technical, or material support: DDB, JD, BG. Steering committee: DDB, SV, EJOK, JD, SV, BG, BVB, EA, RDP.

\section{Compliance with ethical standards}

\section{Conflicts of interest}

DB reports grants from Gilead, Astellas, Fisher-Paykel, Baxter, Alexion and Fresenius Kabi outside the submitted work. KR reports honoraria from Alexion, outside the submitted work. MD reports grant from MSD and Jazz Pharma, personal fees from Astellas and Bristol-Myers Squibb, and non-financial support from Astellas, Bristol-Myers Squibb, Astute Medical, and Sanofi Aventis. EA reports grants and personal fees from Gilead, Alexion, MSD, Cubist and personal fees from Baxter, outside the submitted work. All other authors have no conflict of interest to report.

\section{Open Access}

This article is distributed under the terms of the Creative Commons Attribution-NonCommercial 4.0 International License (http://creativecommons.org/ licenses/by-nc/4.0/), which permits any noncommercial use, distribution, and reproduction in any medium, provided you give appropriate credit to the original author(s) and the source, provide a link to the Creative Commons license, and indicate if changes were made.

Received: 10 February 2018 Accepted: 14 May 2018

Published online: 28 May 2018

\section{References}

1. Wunsch H, Linde-Zwirble WT, Harrison DA, Barnato AE, Rowan KM, Angus DC (2009) Use of intensive care services during terminal hospitalizations in England and the United States. Am J Respir Crit Care Med 180:875-880

2. Teno JM, Gozalo PL, Bynum JPW, Leland NE, Miller SC, Morden NE, Scupp T, Goodman DC, Mor V (2013) Change in end-of-life care for medicare beneficiaries. Site of death, place of care, and health care transtitions in 2001 2005, and 2009. JAMA 309:470-477

3. Ho TH, Barbera L, Saskin R, Lu H, Neville BA, Earle CC (2011) Trends in aggressiveness of end of life cancer care in the universal health care system of Ontario, Canada. J Clin Oncol 29:1587-1591

4. Wang CY, Calfee CS, Paul DW, Janz DR, May AK, Zhuo H, Bernard GR, Matthay MA, Ware LB, Neudoerffer Kangelaris K (2014) One-year mortality and predictors of death among hospital survivors of acute respiratory distress syndrome. Intensive Care Med 40:388-396

5. Garland A, Olafson K, Ramsey CD, Yogendran M, Fransoo R (2014) Distinct determinants of long-term and short-term survival in critical illness. Intensive Care Med 40:1097-1105

6. Kompanje EJ, Piers RD, Benoit DD (2013) Causes and consequences of disproportionate care in intensive care medicine. Curr Opin Crit Care 19:630-635 
7. Bosslet GT, Pope TM, Rubenfeld GD, Lo B, Truog RD, Rushton CH, Curtis JR, ford DW, Osborne M, Misak C, Au DH, Azoulay E, Brody B, Fahy BG, Hall $J B$, Kesecioglu J, Kon AA, Lindell KO, White DB, on behalf of the American Thoracic Society ad hoc committee on Futile and Potentially Inappropriate Treatment (2015) An official ATS/AACN/ACCP/ESICM/SCCM policy statement: responding to requests for potentially inappropriate treatments in intensive care units. Am J Respir Crit Care Med 191:1318-1330

8. Piers R, Azoulay E, Ricou B, Dekeyser Ganz F, Decruyenaere J, Max A, Michalsen A, Azevedo Maia P, Owczuk R, Rubulotta F, Depuydt P, Meert AP, Reyners A, Aquilina A, Bekaert M, Van den Noorgate N, Schrauwen W Benoit D (2011) APPROPRICUS study group of the ESICM. Perception of appropriateness of care among European and Israeli intensive care unit nurses and doctors. JAMA 306:2694-2703

9. Piers R, Azoulay E, Ricou B, Dekeyser Ganz F, Max A, Michalsen A, Azevedo Maia P, Owczuk R, Rubulotta F, Meert AP, Reyners A, Decruyenaere J, Benoit DD (2014) Inappropriate care in European ICUs. Confronting views from nurses and junior and senior physicians. Chest $146: 267-275$

10. Anstey MH, Adams JL, McGlynn EA (2015) Perceptions of the appropriateness of care in California adult intensive care units. Crit Care 19:51

11. Vincent $J L$ (1999) Forgoing life support in western European intensive care units: the results of an ethical questionnaires. Crit Care Med 27:1626-1633

12. Palda VA, Bowman KW, Mclean RF, Chapman MG (2005) "Futile" care: do we provide it? Why? A semistructured, Canada-wide survey of intensive care unit doctors and nurses. J Crit Care 20:207-213

13. Giannini A, Consonni D (2006) Physicians' perceptions and attitudes regarding inappropriate admissions and resource allocation in the intensive care setting. Br J Anaesth 96:57-62

14. Vandenbulcke B, Piers R, Jenssen HI, Malmgrem J, Metaxa V, Reyners AK, Darmon M, Rusinova K, Talmor D, Meert AP, Cancellierre L, Zubek L, Maia P, Michalsen A, Decruyenaere J, Kompanje EJO, Azoulay E, Vlerick P, Van den Sompel A, Vansteelandt S, Vanheule S, Benoit DD (2018) On behalf the DISPROPRICUS study group of the Ethics Section of the ESICM. Ethical decision-making climate in the ICU: theoretical framework and validation of self-assessment tool. BMJ Qual Saf. https://doi.org/10.1136/bmjqs -2017-007390 ([Epub ahead of print])

15. Meadow W, Pohlman A, Reynolds D, Rand L, Correia C, Christoph E, Hall $\mathrm{J}$ (2014) Power and limitations of daily prognostications of death in the medical ICU for outcomes in the following 6 months. Crit Care Med 42:2387-2392

16. Detsky ME, Harhay MO, Bayard DF, Delman AM, Buehler AE, Kent SA Cuiffetelli IV, Cooney E, Gabler NB, Ratcliffe SJ, Mikkelsen ME, Halpern SD (2017) Discriminative accuracy of physician and nurse predictions for survival and functional outcomes 6 months after ICU admission. JAMA 317:2187-2195

17. Singal RK, Sibbald R, Morgan B, Quinlan M, Parry N, Radford M, Martin CM (2014) A prospective determination of the incidence of perceived inappropriate care in critically ill patients. Can Respir J 21:165-170

18. Neville TH, Wiley JF, Yamamoto MC, Flitcraft M, Anderson B, Curtis JR (2015) Concordance of nurses and physicians on whether patients are receiving futile treatment. Am J Crit Care 24:403-411

19. Brislin RW (1976) Translation: application and research. Gardner Press Inc, New York

20. Rhodes A, Ferdinande P, Flaatten H, Guidet B, Metnitz PG, Moreno RP (2012) The variability of critical care bed numbers in Europe. Intensive Care Med 38:1647-1653

21. Rabin R, de Charro F (2001) EQ-5D: a measure of health status from the EuroQol Group. Ann Med 33:337-343
22. Oeyen SG, Vandijck D, Benoit DD, Annemans L, Decruyenaere J (2010) Quality of life after intensive care: a systematic review of the literature. Crit Care Med 38:386-400

23. Everitt BS, Landau S, Leese M, Stahl D (2011) Cluster analyis, 5th edition Wiley. ISBN: 978-0-470-74991-3

24. Putter H, Fiocco M, Geskus RB (2007) Tutorial in biostatistics: competing risks and multi-state models. Statist Med 26:2389-2430

25. Rubin DB (1997) Estimating causal effects from large data sets using propensity scores. Ann Intern Med 127:757-763

26. Lighthall GK, Vazquez-Guillamet C (2015) Understanding decision making in critical care. Clin Med Res 13:156-168

27. Zollo L, Pellegrini MM, Ciappei C (2017) What sparks ethical decision making? The interplay between moral intuition and moral reasoning: lessons from the scholastic doctrine. J Bus Ethics 145:681-700

28. Luce JM, White DB (2009) A history of ethics and law in the intensive care unit. Crit Care Clin 25:221-237

29. Wright AA, Zhang B, Ray A, Mack JW, Trice E, Balboni T, Michell SL, Jackson VA, Block SD, Maciejewski PK, Prigerson HG (2008) Associations between end-of-life discussions, patient mental health, medical care near death, and caregiver bereavement adjustment. JAMA 300:1665-1673

30. Curtis JR, Vincent JL (2010) Ethics and end-of-life care for adults in the intensive care unit. The Lancet 376:1347-1353

31. Kimbell B, Murray SA, Macpherson S, Boyd K (2016) Embracing inherent uncertainty in advanced illness. BMJ 354:3802

32. Dzeng E, Curtis JR (2018) Understanding ethical climate, moral distress, and burnout: a novel tool and a conceptual framework. BMJ Qual Saf:1-5. https://doi.org/10.1136/bmjqs-2018-007905 (Epub ahead of print)

33. Hamric AB, Blackhall $L J$ (2007) Nurse-physician perspective on the care of dying patients in intensive care units: collaboration, moral distress, and ethical climate. Crit Care Med 35(2):422-429

34. Schwarzkopf $D$, Rüddel $H$, Thomas-Rüddel DO, Felfe J, Poidinger $B$, Matthäus-Krämer CT, Hartog CS, Bloos F (2017) Perceived nonbeneficial treatment of patients, burnout, and intention to leave among ICU nurses and junior and senior physicians. Crit Care Med 45:e265-e273

35. Mark NM, Rayner SG, Lee NJ, Curtis JR (2015) Global variability in withholding and withdrawal of life-sustaining treatment in the intensive care unit: a systematic review. Intensive Care Med 41:1572-1585

36. De Angelis R, Sant M, Coleman MP, Francisci S, Baili P, Pierannunzio D, Trama A, Visser O, Brenner H, Ardanaz E, Bielska-Lasota M, Engholm G, Nennecke A, Siesling S, Berrino F, Capocaccia R, Eurocare-5 Working Groups (2014) Cancer survival in Europe 1999-2007 by country and age: results of the Eurocare-5. A population-based study. Lancet Oncol 15:23-34

37. Khandelwal N, Kross EK, Engelberg RA, Coe NB, Long AC, Curtis JR (2015) Estimating the effect of palliative care interventions and advance care planning on the ICU utilization: a systematic review. Crit Care Med 43:1102-1111

38. Puntillo KA, McAdam JL (2006) Communication between physicians and nurses as a target for improving end-of-life care in intensive care unit: challenges and opportunities for moving forward. Crit Care Med 34(Suppl.):S332-S340

39. Jensen HI, Ammentrop J, Erlandsen M, Ording H (2011) Witholding or withdrawing therapy in intensive care units: an analysis of collaboration among healthcare professionals. Intensive Care Med 37:1696-1705

40. Aslakson RA, Curtis JR, Nelson JE (2014) The changing role of palliative care in the ICU. Crit Care Med 42:2418-2428 